\title{
Systemic Analysis of the Dna Replication Regulators Origin Recognition Complex in Lung Adenocarcinomas Identifies Prognostic and Expression Significance
}

\author{
Yun-bo Deng \\ University of South China \\ Juan Chen \\ University of South China \\ Xian-yu Luo \\ University of South China \\ Tian Zeng \\ University of South China \\ Dong-mei Ye \\ The First Affiliated Hospital of nanchang city \\ Yu-kun Li \\ University of south china https://orcid.org/0000-0002-8517-9075 \\ Min Tang \\ University of South China \\ Yu-ping Zhang ( $\nabla$ ypzhang2021@163.com ) \\ University of South China https://orcid.org/0000-0002-9507-5907
}

\section{Primary research}

Keywords: lung adenocarcinomas, ORC complex, public databases, prognostic value, comprehensive bioinformatics

Posted Date: July 8th, 2021

DOI: https://doi.org/10.21203/rs.3.rs-487176/v2

License: (c) (1) This work is licensed under a Creative Commons Attribution 4.0 International License. Read Full License 


\section{Abstract}

Background: Origin recognition complex (ORC) 1, ORC2, ORC3, ORC4, ORC5 and ORC6, form a replicationinitiator complex to mediate DNA replication, which play a key role in carcinogenesis, while their role in lung adenocarcinomas (LUAD) remains poorly understood.

Methods: We confirmed the transcriptional and post-transcriptional levels, DNA alteration, DNA methylation, miRNA network, protein structure, PPI network, functional enrichment, immune infiltration and prognostic value of ORCs in LUAD based on Oncomine, GEPIA, HPA, cBioportal, TCGA, GeneMANIA, Metascape, KM-plot, GENT2, and TIMER database.

Results: ORC mRNA and protein were both enhanced obviously based on Oncomine, Ualcan, GEPIA, TCGA and HPA database. Furthermore, ORC1 and ORC6 have significant prognostic values for LUAD patients based on GEPIA database. Protein structure, PPI network, functional enrichment and immune infiltration analysis indicated that ORC complex cooperatively accelerate the LUAD development by promoting DNA replication, cellular senescence and metabolic process.

Conclusion: the ORC complex has an important prognostic and expression significance for LUAD patients.

\section{Introduction}

Lung adenocarcinomas (LUAD), accounting for $60 \%$ of all lung cancers, is a common type of non-smallcell lung cancer (NSCLC) [1, 2], which has become one of the leading causes of tumor-related mortality worldwide[3]. Despite the development of medical science, the survival rate in advanced-stage cancer remains $57 \%$ for patients with stage I disease and declines to $4 \%$ for those with stage IV disease[4]. Earlystage LUAD are treated with surgery and have a favorable prognosis. In advanced LUAD, there are three main therapy options: chemotherapy, immunotherapy, and targeted therapy[5]. However, some patients with LUAD do not benefit from chemotherapy, immunotherapy, and targeted therapy due to cancerous heterogeneity. It is urgently needed to identify reliable biomarkers as new therapeutic targets for LUAD patients.

The origin recognition complex (ORC) complex, a replication-initiator complex, bind DNA replication origins to activate the initiation of DNA synthesis[6]. ORC proteins can bind to potential origins to formulate pre-replication complex (pre-RC)[7]. The assembly of ORC complex, a heteromeric six-subunit complex, is a highly orchestrated event in eucaryon. Briefly, ORC2, ORC3 and ORC5 form a complex in the cytoplasm, which can transport to cytoblast and bind to ORC4 and ORC6. The five-subunit complex can further bind to ORC1 at the DNA replication origin[8], resulting in the initiation of DNA replication.

ORC proteins are dysregulation and accelerate the formation, development and progression in multiple cancer types. Overexpression of ORC1 mediated by IncRNA XIST/miR-140-5p axis promote the progression of cervical cancer[9]. In hepatocellular carcinoma, ORC1, 5 and 6 are novel biomarkers for the 
diagnosis and prognosis[10]. Previous indicated that polo-like kinase 1 (Plk1) phosphorylation of ORC2 mediates the resistance to gemcitabine in pancreatic cancer[11]. ORC3 can interact with MCM complex to accelerate the progression of hepatocellular carcinoma[12]. ORC4 gene mutation can induce B-cell lymphoproliferative disorders[13]. Moreover, ORC4 can also be a new biomarker for the diagnosis of breast cancer[14]. In HPV-negative head and neck squamous cell carcinoma, ORC5 is a specific biomarker for improving the diagnosis and treatment of patients[15]. Furthermore, ORC2 or ORC5 knockdown can inhibit the recruit of MCM2-7 normally to chromatin in human colon cancer cell lines[16]. ORC6 can be an excellent biomarker for gastric adenocarcinoma, colon cancer, rectal cancer and prostate cancer[17-19]. Inhibiting ORC6 can enhance the sensitizes of 5-fluorouracil and cisplatin in colon cancer patients[20]. These results suggested the important molecular role of ORC complex in the formation, development and progression in multiple cancer types, such as hepatocellular carcinoma, gastric adenocarcinoma, colon cancer, rectal cancer and prostate cancer. Despite of several studies indicated the dysregulation of several ORCs, but the significance of all ORC proteins for prognostic values and targeted treatment in LUAD patients still unclear.

With the establishment of public databases and the development of visual websites, the possibility of systematic analysis of ORC complexes in different tumor types based on bioinformatic analysis are grown up as an important in the field of cancer research. In this study, we systematically confirmed the transcriptional and post-transcriptional level of ORCs and found its prognostic value in LUAD.

Furthermore, we also analyzed the interaction network, DNA alteration, DNA methylation, miRNA network, protein secondary and tertiary structure, Immune infiltration and functional enrichment of ORCs by bioinformatics.

\section{Methods}

\section{The mRNA and protein level of ORCs based on public database}

The TCGA database (https://www.cancer.gov/tcga) included many cancer patients data, such as clinical data, genomic variation, mRNA expression and methylation level expression for multiple cancer types[21]. Oncomine database (https://www.oncomine.org) is used to analyze the ORCs mRNA in multiple cancer types[22]. Furthermore, transcriptional expression analysis using the UALCAN database (http://ualcan.path.uab.edu/index.html)[23]. GEPIA (http://gepia.cancer-pku.cn/) is used in the present study to analyze correlation among ORCs[24]. HPA (http://www.proteinatlas.org) database is an excellent tool for protein level in many cancer types and normal tissues[25]. These public databases were utilized to confirm the mRNA and protein level of ORCs in LUAD.

\section{DNA alteration of ORCs in LUAD}

The association between ORC complex alterations and survival outcome in LUAD patients was confirmed by cBioProtal database (http://www.cbioportal.org/)[26]. This database is an open access, open source resource for interactive exploration of multiple Cancer Genomics datasets. . 


\section{Protein structure analysis for ORC proteins}

Protein Data Bank (PDB) database (https://www.rcsb.org/) is utilized in the structure of protein for ORC complex[27].

\section{Construction for ORC complex Network}

GeneMANIA 3.6.0 (http://www.genemania.org) is used to construct ORC complex network [28]. The max resultant attributes and genes were 10 and 20 , respectively.

\section{GO function enrichment and KEGG pathway}

Metascape (http://metascape.org) is an excellent site for analyzing gene annotations and functional enrichment, which be utilized to make a GO function enrichment and KEGG pathway for ORC proteins and the 20 neighboring genes[29].

\section{Immune Infiltration Analysis for ORC complex}

TIMER (https://cistrome.shinyapps.io/timer/) Is a simple and interactive good online site for analysis of immune infiltration in multiple cancer types[30]. This site was used to further analyze the relationship between immunoinfiltration and the ORC complex.

\section{Survival Analysis}

GENT2 database (http://gent2.appex.kr/gent2/) is a web-site for exploring gene expression patterns by normal and cancer samples, which is used to do a meta-survival analysis[31]. KM-plot database (https://kmplot.com/analysis/) Is able to evaluate the effect of $54 \mathrm{~K}$ genes (mRNA, miRNA, protein) on survival in mutiple cancer types. The sources of the database include GEO, EGA, and TCGA. The main purpose of this tool is to conduct a meta-analysis based on the discovery and validation of survival markers[32].

\section{Clinical samples}

A total of 50 LUAD tissues and 13 normal lung tissues were surgically collected in The Second Affiliated Hospital, University of South China (Hengyang, Hunan, China) from 2015 to 2020. The collection and use of tissues were performed in keeping with the ethics standards as formulated in the Helsinki Declaration. Written informed consent was obtained from each patient, which was approved by the research ethics committee of the University of South China.

\section{IHC staining}

IHC staining was conducted according to our previous report[33].

\section{Statistical Analysis.}


Statistical analyses were performed in the R Programming Language (version 3.6). All statistical tests were bilateral, and $P<0.05$ was statistically significant.

\section{Results}

\section{The transcriptional and post-transcriptional levels of ORCs in LUAD}

The flow diagram of this systemic analysis is shown in Figure 1.

At first, we confirmed these ORC complex transcriptional level in 20 cancer types compared to according normal tissue samples based on oncomine database (Figure 2). Total unique analysis among ORC1, ORC2, ORC3, ORC4, ORC5 and ORC6 were 432, 437, 430, 400, 401 and 369, respectively. These ORCs were both increased in most cancer types, especially in bladder cancer, cervical cancer, colorectal cancer, lung cancer, sarcoma. Moreover, ORC1 mRNA level was enhanced in 17 datasets and reduced in 2 datasets. The transcriptional level of ORC2 was markedly increased in 5 datasets and decreased in 2 datasets. For ORC3, 11 datasets showed upregulated, but 5 datasets showed downregulated. High levels of ORC4 was observed in 2 datasets, and low levels of ORC4 was also observed in 2 datasets. The ORC5 mRNA level was increased in 11 datasets but decreased in 4 datasets. At last but not least, ORC6 was significantly increased in 36 datasets, but decreased in 3 datasets.

Subsequently, we confirmed the transcriptional level of ORC complex in LUAD patients based on UALCAN database. The results indicated that both ORC mRNA levels were significantly enhanced in LUAD patients compared to normal lung tissue samples (Figure 3A). The survival analysis data among these ORC complexes was also showed that the mRNA levels of ORC1 and ORC6 was significantly and negatively correlated with prognosis in LUAD patients. However, the mRNA levels of other ORCs were not correlated the LUAD patient's prognosis (Figure 3B).

Then, we extracted the post-transcriptional level among ORCs in LUAD patients based on HPA database. The results showed that the IHC staining intensity of ORCs were obviously and significantly increased in LUAD tissue samples compared to normal lung tissue samples (Figure 4A). The survival analysis data based on ORCs protein expression showed that ORC1, ORC5 and ORC6 were negatively and significantly correlated with prognosis in LUAD patients. But the ORC2, ORC3 and ORC4 was not significantly correlated with LUAD patient's prognosis (Figure 4B). We further confirmed the expression of ORCs by IHC staining in LUAD samples and normal lung samples, which showed that ORC1-6 expressions were obviously increased in the cancer tissues of 50 LUAD patients compared with 13 normal lung samples (Figure 5A). Furthermore, our results also suggested that ORC1/6 expression negatively associated with the overall survival (OS) rate (Figure 5B). The difference between our result and HPA results might be attributed to the difference in sample size.

\section{Prognostic Values of ORC complex in LUAD Patients}


From the above analysis, we found that the prognostic values of these ORCs were obviously different in each other based on the transcriptional and post-transcriptional levels. To further validate the prognostic value of these ORCs, we conducted survival analyses of the ORCs used by KM-plot database and GEO database. The meta-survival analyses showed that ORC1, ORC2, ORC5 and ORC6 had a prognostic value (Figure 5A). KM-plot database analysis showing overall survival probability of ORC1, ORC3 and ORC6 were significant (Figure 5B). Taken together, the prognostic values of ORC1 and ORC6 is more significant compared to other ORCs in LUAD patients.

\section{The underlying mechanism of the ORC complex regulation based on bioinformatic analysis}

In DNA level, we explored the alteration level in ORC complex based on cBioProtal database. The lung cancer dataset was showed that the DNA alteration percentages of ORC complex were $1.9 \%$ (ORC1), $1.7 \%$ (ORC2), 1.5\% (ORC3), 1.2\% (ORC4), 2.2\% (ORC5), and 1.4\% (ORC6), respectively (Figure 6A-B). Then, we further confirmed the survival rate between ORC complex alteration group and no alteration group (Figure $6 C$ ), which indicated that the DNA alteration was not correlated with the prognosis outcome in LUAD patients. Moreover, we also investigated the DNA methylation level of ORCs in LUAD based on TCGA database. The result indicated that the methylated level was significantly decreased in the CpG promoter of ORC1 and ORC2, but significantly increased in the CpG promoter of ORC4 for LUAD tissue samples compared to normal lung tissue samples (Table 1 \& Figure 7A). However, the Heat map with hierarchical clustering of $\mathrm{CpG}$ methylation among ORCs has no significance (Figure 7B), which indicated that methylation profiles cannot be used to distinguish normal samples from cancers. These results indicated that these ORCs were regulated by DNA methylation rather than DNA alteration in DNA level.

In mRNA level, we extracted the profiles of miRNA interacted ORC complex based on GSCALite database. The results showed that ORC1 and ORC2 could be regulated by multiple miRNAs, including hsa-miR-3132, hsa-miR-125b-5p, hsa-miR-7-5p, hsa-miR-200a-3p, hsa-miR-375, hsa-miR-330-5p, hsa-miR-539-5p, hsamiR-125a-5p, hsa-miR-141-3p, hsa-miR-143-3p, hsa-miR-183-5p, hsa-miR-636, hsa-miR-24-3p, hsa-miR1224-3p, hsa-miR-149-5p, hsa-miR-2110, hsa-miR-4254, hsa-miR-1254, hsa-miR-124-3p, hsa-miR-671-5p, hsa-miR-150-5p for ORC1 , and hsa-miR-122-5p, hsa-miR-3154, hsa-miR-15b-5p, hsa-miR-491-3p, hsa-miR486-5p for ORC2 (Figure 7C). There results indicated that ORC1 and ORC2 might be regulated by many miRNAs in mRNA level, resulting in dysregulation of ORC complex.

In protein level, we investigated the protein secondary structure data in ORCs based on PDB database (Figure 8). ORC1 has three classic domains, such as BAH, AAA, and Cdc6_C. ORC1 also had several chemical modification types (phosphirylation, acetylation, ubiquitination and methylation). ORC2 has ORC2 domain, and two chemical modification types (phosphirylation and acetylation). ORC3 has AAA_16 domain and four chemical modification types (phosphirylation, acetylation, ubiquitination and glutathionylation). ORC4 has two domains, such as AAA_16 and ORC4_C domain, and may regulated by phosphirylation, acetylation, ubiquitination and methylation. ORC5, modified by phosphirylation, acetylation and ubiquitination, has AAA_16 and ORC5_C domain. ORC6 has a ORC6 domain, and can be 
regulated by phosphirylation, acetylation, ubiquitination, methylation and sumoylation. Taken together, these results indicated that the activity of these ORCs could be mediated by multiple chemical modification types.

\section{Functional enrichment and pathway analyses for ORC complex in LUAD}

Subsequently, the structural models of six ORC subunits were also constructed by PDB database (Figure $9 \mathrm{~A})$, which indicated that these ORCs were able to bind each other. We also excavated the expression data of ORCs in LUAD patients to analysis the correlations among these ORC complex (Figure 9B). This result indicated a positively and significantly correlation between these ORC1/2/3/4/5/6 and other ORCs, especially in ORC1-ORC6 $(R=0.66)$ and ORC2-ORC4 $(R=0.54)$. Next, the protein-protein interaction networks of ORC complex were constructed by GeneMANIA, including ORC1, ORC2, ORC3, ORC4, ORC5, ORC6, LRWD1, DBF4, HMGA1, HIST1H3I, CDC6, MCM5, MCM4, CDC45, MCM7, CDC7, MCM2, MCM3, MCM6, MCM10, TERF2, HIST4H4, MCM8, CBX5, CDT1 and CCNE2 (Figure 9C). Then, we used these genes generated by GeneMANIA tools to further analyze the GO functional enrichment and KEGG pathway analyses by Metascape database. Pathway and process enrichment analysis indicated that these genes had an important effect on activation of the pre-replicative complex, regulation of nuclear cell cycle DNA replication, regulation of chromosome organization, PID E2F pathway, cellular senescence, PID ATR pathway, and 22q11.2 copy number variation syndrome (Figure 9D). The top-level Gene Ontology biological processes showed that these genes were enriched in multiple biological progression, such as metabolic process, cellular process, response to stimulus, cell component organization or biogenesis, regulation of biological process, positive regulation of biological process, negative regulation of biological process, localization, and biological regulation (Figure 9E). Then, we also constructed networks for pathway and process enrichment analysis (Figure 9F) and protein-protein interaction enrichment analysis (Figure 9G), which showed the interaction among these GO enrichments and KEGG pathway analysis.

\section{The correlation between ORC complex expression and immune infiltration}

Owing to the significant role of tumor-infiltrating immune in carcinogenesis and its impact on prognosis, we also confirmed the immune infiltration of ORC complex in LUAD based on the GEPIA database. Our result indicated that the expression of ORC1, ORC2, ORC3, ORC4, ORC5 and ORC6 had a different level in the seven-common tumor-infiltrating immune cells, such as B cells, CD4 T cells, CD8 T cells, NK cells, macrophages, endothelials, and cancer associated fibroblasts (Figure 10A). Furthermore, we found ORC1 had a correlation with purity $(p=2.16 \mathrm{e}-03$, cor $=0.138), B$ cell $(p=1.31 \mathrm{e}-06$, cor $=-0.218), C D 8^{+} T$ cell $(p=7.87 e-01$, cor $=-0.218), C D 4^{+} T$ cell $(p=2.99 e-04$, cor $=-0.164)$, Macrophage $(p=1.69 e-01$, cor $=-0.063)$, Neutrophil ( $p=3.16 \mathrm{e}-03$, cor $=-0.134)$, and Dendritic cell $(p=3.72 e-08$, cor $=-0.246)$. ORC2 expression was associated with purity ( $p=7.43 e-02$, cor $=0.08)$, B cell $(p=1.07 e-01$, cor $=-0.073), C D 8^{+} T$ cell $(p=4.45 e-07$, cor=0.226), $C^{+} 4^{+} T$ cell $(p=5.77 e-01, c o r=0.025)$, Macrophage $(p=2.24 e-01$, cor $=0.055)$, Neutrophil $(p=9.39 \mathrm{e}-05$, cor $=0.177)$, and Dendritic cell $(p=7.30 \mathrm{e}-01$, cor $=0.016)$. The expression of ORC3 was correlated with purity $(p=4.96 e-01$, cor $=-0.031), B$ cell $(p=1.15 e-01$, cor $=0.072), C^{+} 8^{+}$cell $(p=5.29 e-03$, 
cor $=0.126), C D 4^{+} T$ cell $(p=2.83 e-02$, cor $=-0.1)$, Macrophage $(p=2.51 e-02$, cor=0.102), Neutrophil $(p=2.19 e-$ 02 , cor $=0.104)$, and Dendritic cell $(p=8.06 \mathrm{e}-05$, cor $=0.178)$. The ORC4 mRNA level was correlated with purity $(p=7.4 \mathrm{e}-03$, cor $=0.12), B$ cell $(p=8.24 \mathrm{e}-02$, cor $=-0.079), C^{+} \mathrm{T}$ cell $(p=1.86 \mathrm{e}-04$, cor $=0.169), \mathrm{CD}^{+} \mathrm{T}$ cell $(p=7.59 e-03$, cor $=-0.121)$, Macrophage $(p=4.54 e-02$, cor $=0.091)$, Neutrophil $(p=8.74 e-04$, cor $=0.151)$, and Dendritic cell $(p=6.15 \mathrm{e}-01$, cor $=0.023)$. ORC5 was correlated with purity $(p=7.4 \mathrm{e}-01$, cor $=0.015)$, B cell $(p=2 e-04$, cor $=-0.168), C D 8^{+} T$ cell $\left(p=4.48 \mathrm{e}-03\right.$, cor=0.129), $C^{+} 4^{+} T$ cell $(p=1.70 e-04, c o r=-0.17)$, Macrophage ( $p=7.48 \mathrm{e}-01$, cor $=-0.015)$, Neutrophil $(p=2.84 \mathrm{e}-03$, cor $=0.136)$, and Dendritic cell $(p=6.33 \mathrm{e}-01$, cor=0.022). ORC6 was also correlated with purity ( $p=7.08 \mathrm{e}-01$, cor $=0.017)$, B cell $(p=3.41 e-04, c o r=-0.162)$, $\mathrm{CD}^{+} \mathrm{T}$ cell $(p=6.35 \mathrm{e}-01, \mathrm{cor}=-0.022), \mathrm{CD}^{+} \mathrm{T}$ cell $(p=6.80 \mathrm{e}-03, \mathrm{cor}=-0.123)$, Macrophage $(p=3.25 \mathrm{e}-03$, cor=-0.133), Neutrophil ( $p=4.01 \mathrm{e}-01$, cor=0.038), and Dendritic cell $(p=2.60 \mathrm{e}-02$, cor $=-0.101)$ (Figure 10B). Therefore, these ORCs were closely associated with immune infiltration.

\section{Verification of the drug sensitivity of hub ORC proteins}

Finally, we analyzed the drug sensitivity of hub ORC proteins. Our results indicated that ORC1 (SLC25A15) and ORC2 (SLC25A2) was closely associated with chemotherapy resistance based on the GSCALite database (Supplementary Figures S1 and S2). Hence, these results indicated that ORC1 and ORC2 could be potential therapeutic target for LUAD patients.

\section{Discussion}

It has already been reported that ORC complex can promote the initiation of DNA synthesis[6, 34], but there are no much data in the literature on interaction with cancer development and progression. In previous studies, the heterohexameric complex composed of ORC1-6 has been systematically analyzed in hepatocellular carcinoma[6]. Nevertheless, little is known about the prognostic and expression significance of ORC1-6 in LUAD. Hence, our study conducted a comprehensive and systematic analysis to elucidate the transcriptional level, post-transcriptional level, protein structure, chemical modification, function enrichment, protein interaction, prognostic values, immune infiltration and drug sensitivity of ORC proteins in LUAD.

In this study, we found that ORC proteins were both significantly increased in LUAD tissues samples compared to normal lung tissues samples at the transcriptional and post-transcriptional level. Yitong Zhang et al. indicated that ORC1 level increased according to the pathological stages of LUAD, which could be utilized as a therapeutic target in LUAD treatment, by inhibiting the stemness features[35]. Susana Gonzalez and his colleagues indicated that repression of the INK4/ARF locus could suppress the oncogenic activity of Cdc6 in lung cancer, which could inhibit the formation of multiprotein complex, including ORC2, Cdc6 and MCMs[36]. these studies indicated ORC1 and ORC2 might be a potential oncogene in the development and progression of lung cancer. Regrettably, it has been largely overlooked how ORC3/4/5/6 and LUAD cells perform crosstalk using molecular-based language. 
A large number of studies have identified ORC complex, as a replication-initiator complex, can bind to Cdc6 and Cdt1, which can ultimately combine with the loading of two MCM complex. The MCM2-7 double hexamer promotes the DNA replication at the replication origin. Moreover, the molecular of the DNA synthesis by ORC-Cdc6-Cdt1-MCM complex is highly conserved, especially in mammalian species and cell types[34]. In conjunction with our protein tertiary structure of results, the ORC proteins can bind each other to form a hexamer. The level of six ORC proteins were both enhanced significantly, and highly correlated with each other in LUAD. Taken together, these results indicated that ORC complex might promote the DNA replication in the LUAD progression.

In our functional enrichment and pathway analyses, besides the regulation of nuclear cell cycle DNA replication which have been reported by many studies, ORC proteins also showed other important molecular biological effects, such as cellular senescence and metabolic process. There is recent literature suggesting that ORC1 can activate ERK and JNK signaling pathway to enhance the proliferation and metastasis ability in glioma[37]. Junsuo Kan et al. also found that ORC complex can collaborate with SPP1 to upregulate H3K4me3 in saccharomyces cerevisiae. Due to ORC complex is functionally highly conserved between yeast and humans, the effect of ORC complex on histone methylation are highly similar[38]. In summary, these results suggested ORC proteins not only participated in DNA replication, but also has other molecular functions.

It is interesting to note that the enhanced dependence of the ORC mutant cells on CDC6 for viability drives it likely that CDC6, perhaps with ORC6, can under exceptional circumstances carry out the function of the ORC ring in recruitment and loading of the MCM2-7 pre-helicase complex around DNA[16]. ORC5 is frequently deleted in acute myeloid leukemia (AML) and myelodysplastic syndrome (MDS), which is appeared to be a candidate tumor suppressor gene for these diseases. But ORC5 is not function as a tumor suppressor in these diseases, thus implying the DNA alteration has a little effect on ORC5[39]. We also confirmed the DNA alteration in ORC proteins. The DNA alteration of ORC1/2/3/4/5/6 were $1.9 \%$, $1.7 \%, 1.5 \%, 1.2 \%, 2.2 \%$ and $1.4 \%$, respectively. However, the ORC alteration is not significantly correlated with the prognosis for LUAD patients.

We found the expression of ORC levels might be regulated by CpG methylation at DNA level, miRNA regulation at mRNA level, and chemical modification at protein level. To date, no literature has previously reported these regulations of ORC complex in LUAD, which also indicate the future research directions.

There are several limitations for this study. At first, our study was based on multiple public databases, which was required to further verify the expression of these ORCs in LUAD cell lines and human LUAD samples. Then, the potential molecular mechanisms (apart from DNA replication), especially in cellular senescence and metabolic process, was needed to be further clarify in additional experiments. These results will help to elucidate the role of ORCs and relevant signaling pathways in the formation, development and progression of LUAD.

\section{Conclusion}


In our study, we systematically and comprehensively concluded the transcriptional and posttranscriptional ORC complex and their prognostic significance in LUAD. We also confirmed the regulation of ORCs at DNA level (DNA alteration and methylation), mRNA level (miRNA regulation), and protein level (chemical modification). Furthermore, we analyzed co-expression, interaction network, structural models, enrichment pathways, immune infiltration and drug sensitivity of ORC proteins. The results showed that the transcriptional and post-transcriptional ORC complex was significantly increased in LUAD samples compared to normal samples. The mRNA and protein levels of ORC1 and ORC6 are significantly correlated with LUAD prognosis. Moreover, apart from DNA replication, ORC complex may have other important biological functions, such as immune infiltration, cellular senescence and metabolic process. In summary, ORCs can be new biomarkers with prognostic and expression significance in LUAD patients, which will help develop more valid clinical treatment in the future.

\section{Declarations}

\section{Ethics approval and consent to participate}

Not applicable.

\section{Consent for publication}

Not applicable.

\section{Availability of data and materials}

The datasets presented in this study can be found in online repositories. The names of the repository/repositories and accession number(s) can be found in the article/supplementary material.

\section{Competing interests}

The authors declare that the research was conducted in the absence of any commercial or financial relationships that could be construed as a potential conflict of interest.

\section{FUNDING}

The present study was supported by the Hunan Provincial Health Commission 2019 Annual Scientific Research (Grant No. C2019098).

\section{AUTHOR CONTRIBUTIONS}

M Tang, J Zou and T Zeng, analyzed the data. DM Ye, QH Hu and XX Hu used online tools. X Liu and YK Li designed the project, selected the analyzed results, and wrote the paper. All authors contributed to the article and approved the submitted version

\section{ACKNOWLEDGMENTS}


The authors would like to thank the GEPIA, UALCAN, Oncomine, cBioProtal, PDB, TIMER, DAVID, GTEX and TCGA databases for the availability of the data. We thank Prof. Dai-chao Wu, University of Maryland Institute for Bioscience and Biotechnology Research, Rockville, MD 20850, USA, for their comments on the manuscript.

\section{References}

1. Behera M, Owonikoko TK, Gal AA, Steuer CE, Kim S, Pillai RN, Khuri FR, Ramalingam SS, Sica GL. Lung Adenocarcinoma Staging Using the 2011 IASLC/ATS/ERS Classification: A Pooled Analysis of Adenocarcinoma In Situ and Minimally Invasive Adenocarcinoma. Clin Lung Cancer. 2016;17:e5764.

2. Munkhbaatar E, Dietzen M, Agrawal D, Anton M, Jesinghaus M, Boxberg M, Pfarr N, Bidola P, Uhrig S, Höckendorf U, Meinhardt AL, Wahida A, Heid I, Braren R, Mishra R, Warth A, Muley T, Poh PSP, Wang X, Fröhling S, Steiger K, Slotta-Huspenina J, van Griensven M, Pfeiffer F, Lange S, Rad R, Spella M, Stathopoulos GT, Ruland J, Bassermann F, Weichert W, Strasser A, Branca C, Heikenwalder M, Swanton C, McGranahan N, Jost PJ. MCL-1 gains occur with high frequency in lung adenocarcinoma and can be targeted therapeutically. Nat Commun. 2020;11:4527.

3. Chen Y, Hou C, Zhao LX, Cai QC, Zhang Y, Li DL, Tang Y, Liu HY, Liu YY, Zhang YY, Yang YK, Gao CW, Yao Q, Zhu QS, Cao CH. The Association of microRNA-34a With High Incidence and Metastasis of Lung Cancer in Gejiu and Xuanwei Yunnan. Front Oncol. 2021;11:619346.

4. Miller KD, Nogueira L, Mariotto AB, Rowland JH, Yabroff KR, Alfano CM, Jemal A, Kramer JL, Siegel RL. Cancer treatment and survivorship statistics, 2019. CA Cancer J Clin. 2019;69:363-85.

5. Mok TSK, Wu YL, Kudaba I, Kowalski DM, Cho BC, Turna HZ, Castro G Jr, Srimuninnimit V, Laktionov KK, Bondarenko I, Kubota K, Lubiniecki GM, Zhang J, Kush D, Lopes G. Pembrolizumab versus chemotherapy for previously untreated, PD-L1-expressing, locally advanced or metastatic non-smallcell lung cancer (KEYNOTE-042): a randomised, open-label, controlled, phase 3 trial. Lancet. 2019;393:1819-30.

6. Ortega MA, Nguyen H, Ward WS. ORC proteins in the mammalian zygote. Cell Tissue Res. 2016;363:195-200.

7. Ticau S, Friedman LJ, Ivica NA, Gelles J, Bell SP. Single-molecule studies of origin licensing reveal mechanisms ensuring bidirectional helicase loading. Cell. 2015;161:513-25.

8. Ghosh S, Vassilev AP, Zhang J, Zhao Y, DePamphilis ML. Assembly of the human origin recognition complex occurs through independent nuclear localization of its components. J Biol Chem. 2011;286:23831-41.

9. Chen X, Xiong D, Ye L, Wang K, Huang L, Mei S, Wu J, Chen S, Lai X, Zheng L, Wang M. Up-regulated IncRNA XIST contributes to progression of cervical cancer via regulating miR-140-5p and ORC1. Cancer Cell Int. 2019;19:45.

10. Wang XK, Wang QQ, Huang JL, Zhang LB, Zhou X, Liu JQ, Chen ZJ, Liao XW, Huang R, Yang CK, Zhu GZ, Han CY, Ye XP, Peng T. Novel candidate biomarkers of origin recognition complex 1, 5 and 6 for 
survival surveillance in patients with hepatocellular carcinoma. J Cancer. 2020;11:1869-82.

11. Song B, Liu XS, Rice SJ, Kuang S, Elzey BD, Konieczny SF, Ratliff TL, Hazbun T, Chiorean EG, Liu X. Plk1 phosphorylation of orc2 and hbo1 contributes to gemcitabine resistance in pancreatic cancer. Mol Cancer Ther. 2013;12:58-68.

12. Cao T, Yi SJ, Wang LX, Zhao JX, Xiao J, Xie N, Zeng Z, Han Q, Tang HO, Li YK, Zou J, Wu Q, Identification of the DNA Replication Regulator MCM Complex Expression and Prognostic Significance in Hepatic Carcinoma, Biomed Res Int, 2020 (2020) 3574261.

13. Radojkovic M, Ristic S, Divac A, Tomic B, Nestorovic A, Radojkovic D. Novel ORC4L gene mutation in B-cell lymphoproliferative disorders. Am J Med Sci. 2009;338:527-9.

14. Willmore-Payne C, Damjanovich-Colmenares K, Pasi AV, Werner TL, Gulbahce HE, Downs-Kelly E, Geiersbach KB. Inconsistent Results With Different Secondary Reflex Assays for Resolving HER2 Status. Am J Clin Pathol. 2016;146:618-26.

15. Su Y, Zeng Z, Rong D, Yang Y, Wu B, Cao Y. PSMC2, ORC5 and KRTDAP are specific biomarkers for HPV-negative head and neck squamous cell carcinoma. Oncol Lett. 2021;21:289.

16. Shibata E, Dutta A. A human cancer cell line initiates DNA replication normally in the absence of ORC5 and ORC2 proteins. J Biol Chem. 2020;295:16949-59.

17. Mao R, Wang Z, Zhang Y, Chen Y, Liu Q, Zhang T, Liu Y. Development and validation of a novel prognostic signature in gastric adenocarcinoma. Aging. 2020;12:22233-52.

18. Wei J, Yin Y, Deng Q, Zhou J, Wang Y, Yin G, Yang J, Tang Y. Integrative Analysis of MicroRNA and Gene Interactions for Revealing Candidate Signatures in Prostate Cancer. Front Genet. 2020;11:176.

19. Qi X, Lin Y, Liu X, Chen J, Shen B. Biomarker Discovery for the Carcinogenic Heterogeneity Between Colon and Rectal Cancers Based on IncRNA-Associated ceRNA Network Analysis. Front Oncol. 2020;10:535985.

20. Gavin EJ, Song B, Wang Y, Xi Y, Ju J. Reduction of Orc6 expression sensitizes human colon cancer cells to 5-fluorouracil and cisplatin. PLoS One. 2008;3:e4054.

21. Tomczak K, Czerwinska P, Wiznerowicz M. The Cancer Genome Atlas (TCGA): an immeasurable source of knowledge. Contemp Oncol (Pozn). 2015;19:A68-77.

22. Rhodes DR, Yu J, Shanker K, Deshpande N, Varambally R, Ghosh D, Barrette T, Pandey A, Chinnaiyan AM. ONCOMINE: a cancer microarray database and integrated data-mining platform. Neoplasia. 2004;6:1-6.

23. Chandrashekar DS, Bashel B, Balasubramanya SAH, Creighton CJ, Ponce-Rodriguez I, Chakravarthi B, Varambally S, UALCAN: A Portal for Facilitating Tumor Subgroup Gene Expression and Survival Analyses, Neoplasia, 19 (2017) 649-658.

24. Tang Z, Li C, Kang B, Gao G, Li C, Zhang Z. GEPIA: a web server for cancer and normal gene expression profiling and interactive analyses. Nucleic Acids Res. 2017;45:W98-w102.

25. Uhlen M, Zhang C, Lee S, Sjostedt E, Fagerberg L, Bidkhori G, Benfeitas R, Arif M, Liu Z, Edfors F, Sanli K, von Feilitzen K, Oksvold P, Lundberg E, Hober S, Nilsson P, Mattsson J, Schwenk JM, Brunnstrom H, 
Glimelius B, Sjoblom T, Edqvist PH, Djureinovic D, Micke P, Lindskog C, Mardinoglu A, Ponten F. A pathology atlas of the human cancer transcriptome, Science, 357 (2017).

26. Cerami E, Gao J, Dogrusoz U, Gross BE, Sumer SO, Aksoy BA, Jacobsen A, Byrne CJ, Heuer ML, Larsson E, Antipin Y, Reva B, Goldberg AP, Sander C, Schultz N. The cBio cancer genomics portal: an open platform for exploring multidimensional cancer genomics data. Cancer Discov. 2012;2:401-4.

27. Ormö M, Cubitt AB, Kallio K, Gross LA, Tsien RY, Remington SJ. Crystal structure of the Aequorea victoria green fluorescent protein. Science. 1996;273:1392-5.

28. Warde-Farley D, Donaldson SL, Comes O, Zuberi K, Badrawi R, Chao P, Franz M, Grouios C, Kazi F, Lopes CT, Maitland A, Mostafavi S, Montojo J, Shao Q, Wright G, Bader GD, Morris Q. The GeneMANIA prediction server: biological network integration for gene prioritization and predicting gene function. Nucleic Acids Res. 2010;38:W214-20.

29. Zhou Y, Zhou B, Pache L, Chang M, Khodabakhshi AH, Tanaseichuk O, Benner C, Chanda SK. Metascape provides a biologist-oriented resource for the analysis of systems-level datasets. Nat Commun. 2019;10:1523.

30. Li T, Fu J, Zeng Z, Cohen D, Li J, Chen Q, Li B, Liu XS. TIMER2.0 for analysis of tumor-infiltrating immune cells. Nucleic Acids Res. 2020;48:W509-w514.

31. Park SJ, Yoon BH, Kim SK, Kim SY. GENT2: an updated gene expression database for normal and tumor tissues. BMC Med Genom. 2019;12:101.

32. Nagy Á, Munkácsy G, Győrffy B. Pancancer survival analysis of cancer hallmark genes. Scientific reports. 2021;11:6047.

33. Tang M, Li Y, Luo X, Xiao J, Wang J, Zeng X, Hu Q, Chen X, Tan SJ, Hu J. Identification of Biomarkers Related to CD8(+) T Cell Infiltration With Gene Co-expression Network in Lung Squamous Cell Carcinoma. Front Cell Dev Biol. 2021;9:606106.

34. Kang S, Kang MS, Ryu E, Myung K. Eukaryotic DNA replication: Orchestrated action of multi-subunit protein complexes. Mutat Res. 2018;809:58-69.

35. Zhang Y, Tseng JT, Lien IC, Li F, Wu W, Li H, mRNAsi Index: Machine Learning in Mining Lung Adenocarcinoma Stem Cell Biomarkers, Genes (Basel), 11 (2020).

36. Gonzalez S, Serrano M. A new mechanism of inactivation of the INK4/ARF locus. Cell Cycle. 2006;5:1382-4.

37. Xiong W, Xie C, Qiu Y, Tu Z, Gong Q. Origin recognition complex subunit 1 regulates cell growth and metastasis in glioma by altering activation of ERK and JNK signaling pathway. Mol Cell Probes. 2020;49:101496.

38. Kan J, Zou L, Zhang J, Wu R, Wang Z, Liang C. Origin recognition complex (ORC) mediates histone 3 lysine 4 methylation through cooperation with Spp1 in Saccharomyces cerevisiae. J Biol Chem. 2008;283:33803-7.

39. Fröhling S, Nakabayashi K, Scherer SW, Döhner H, Döhner K. Mutation analysis of the origin recognition complex subunit 5 (ORC5L) gene in adult patients with myeloid leukemias exhibiting deletions of chromosome band 7q22. Hum Genet. 2001;108:304-9. 
Figures

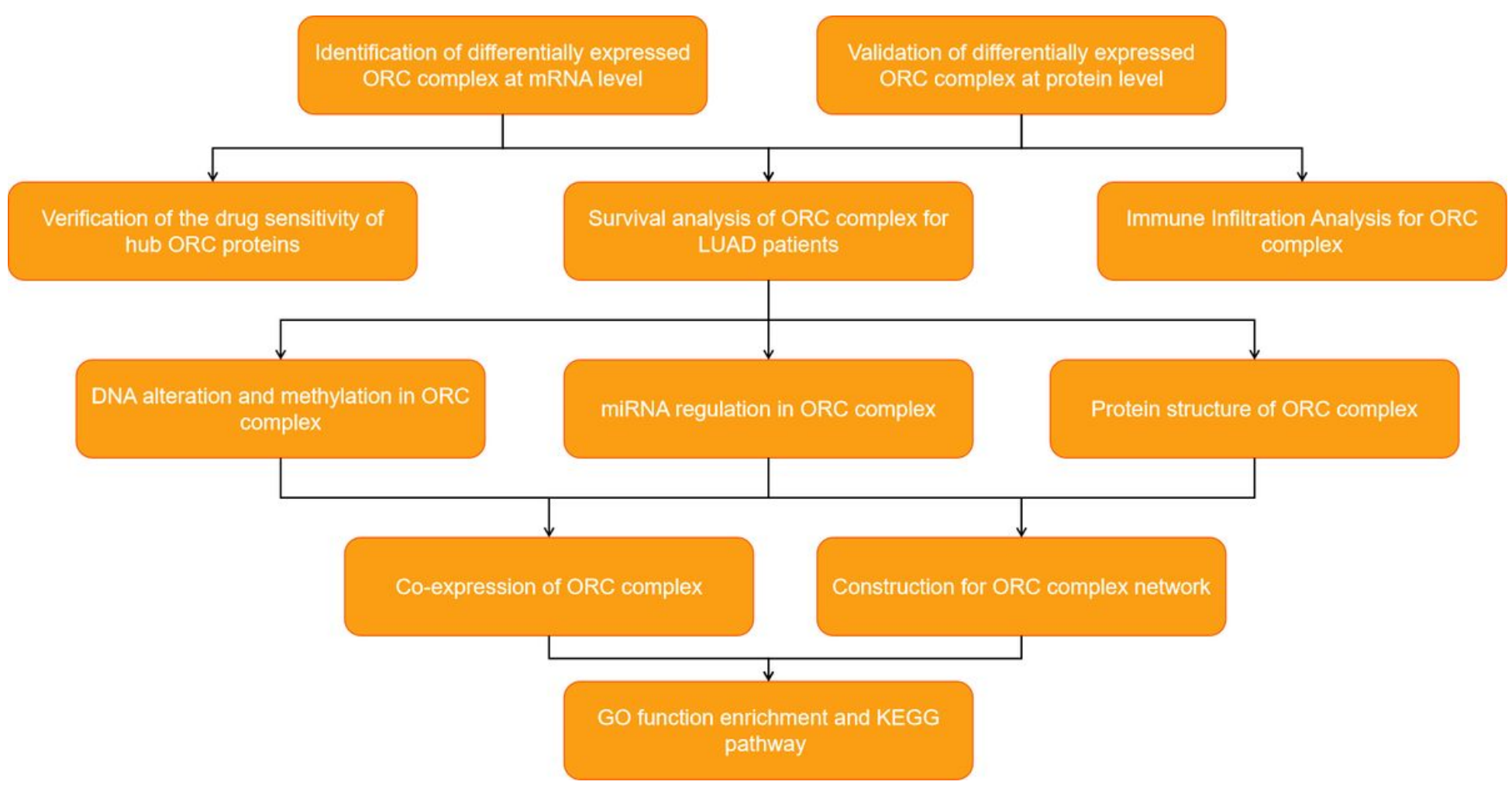

\section{Figure 1}

The work flow of the study 


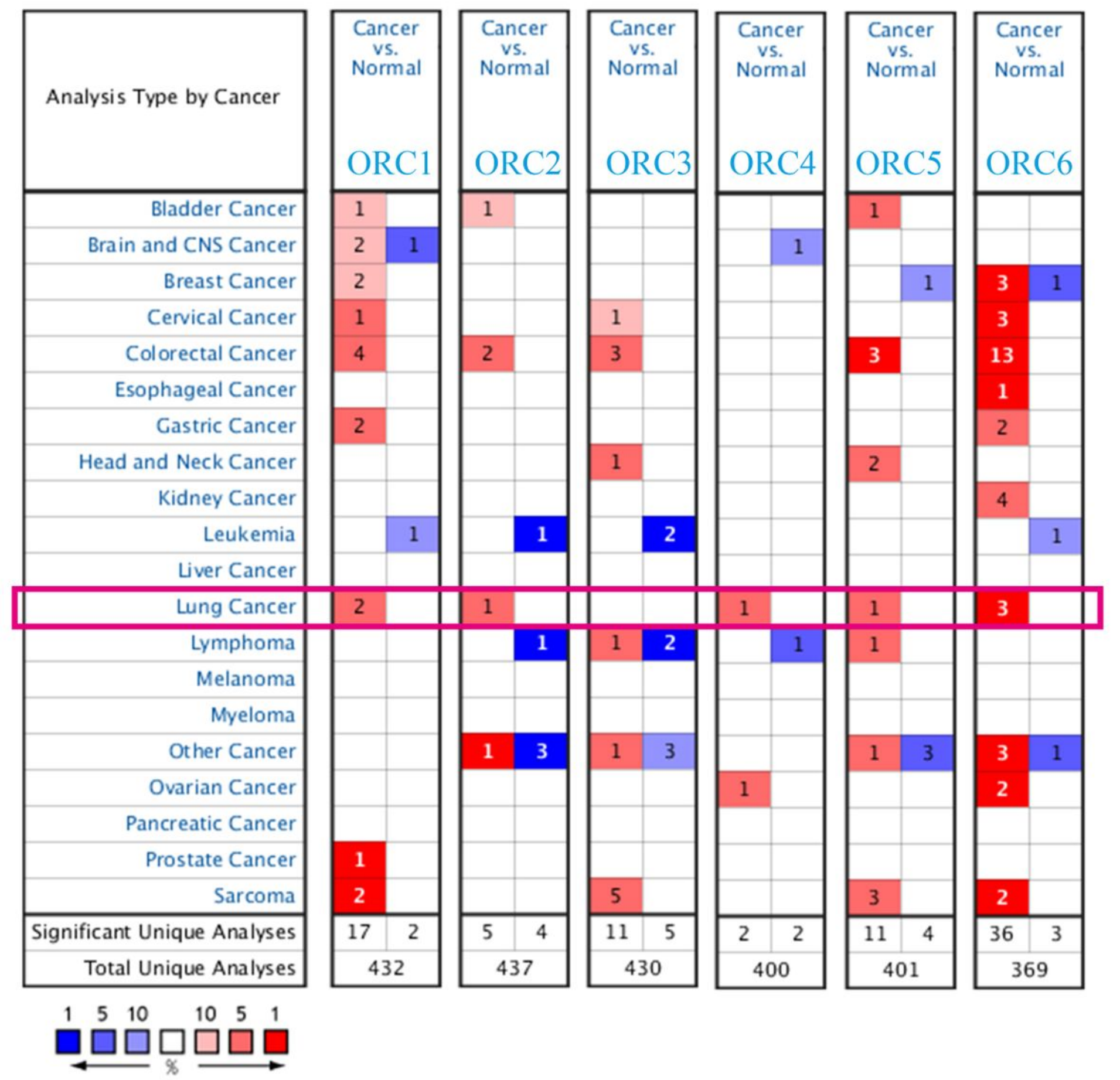

Figure 2

mRNA level of ORC members in 20 cancers types. ORCs level in 20 cancers. Red color suggested the gene is enhanced in cancer samples compared to normal samples, whereas blue color has a reverse meaning to red color. The color depth is FDR. (P value $\leq 0.05 ;|F D R| \geq 2$; gene rank $\leq 10 \%$; data type: $m R N A$ ) 

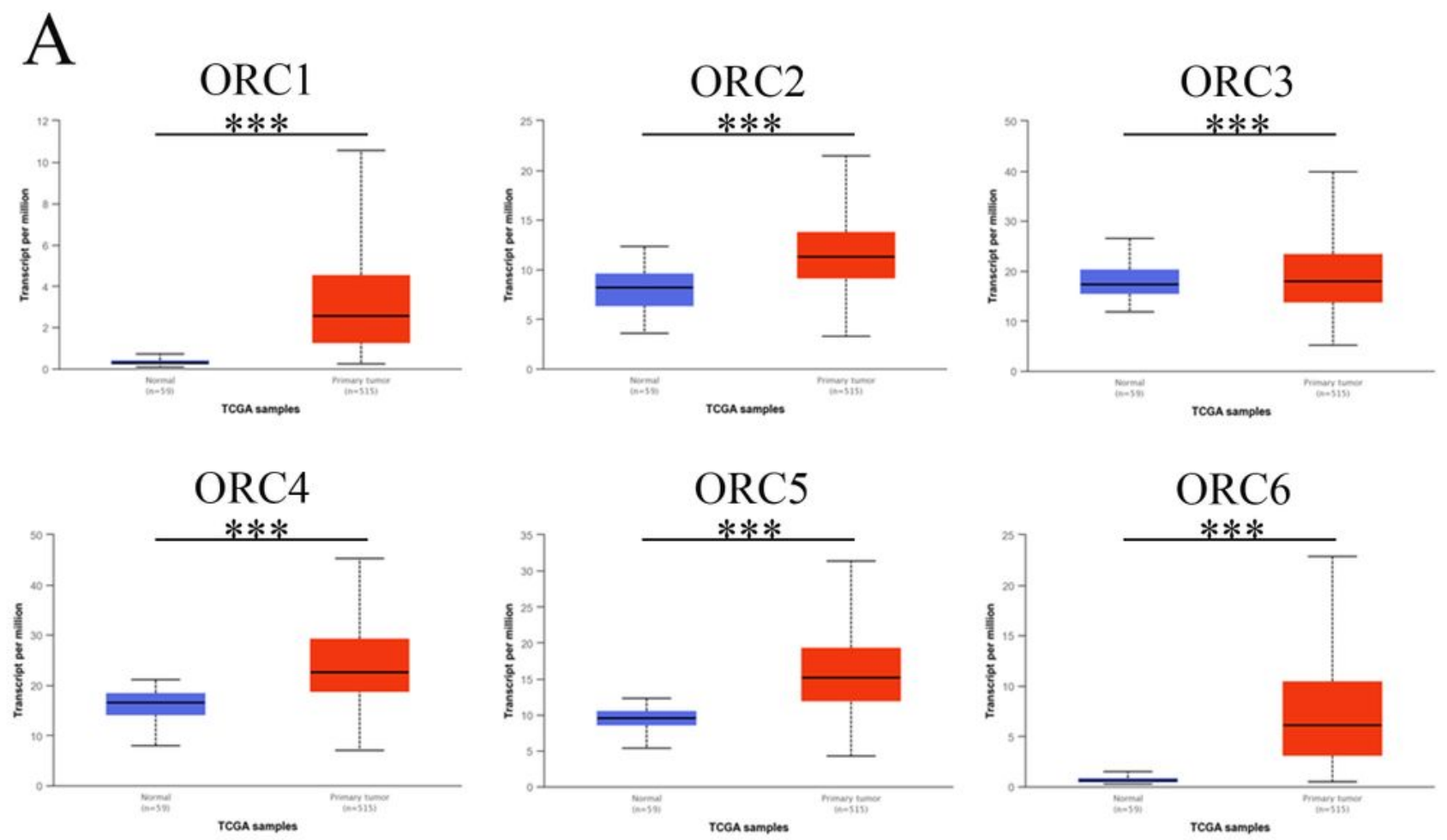

B
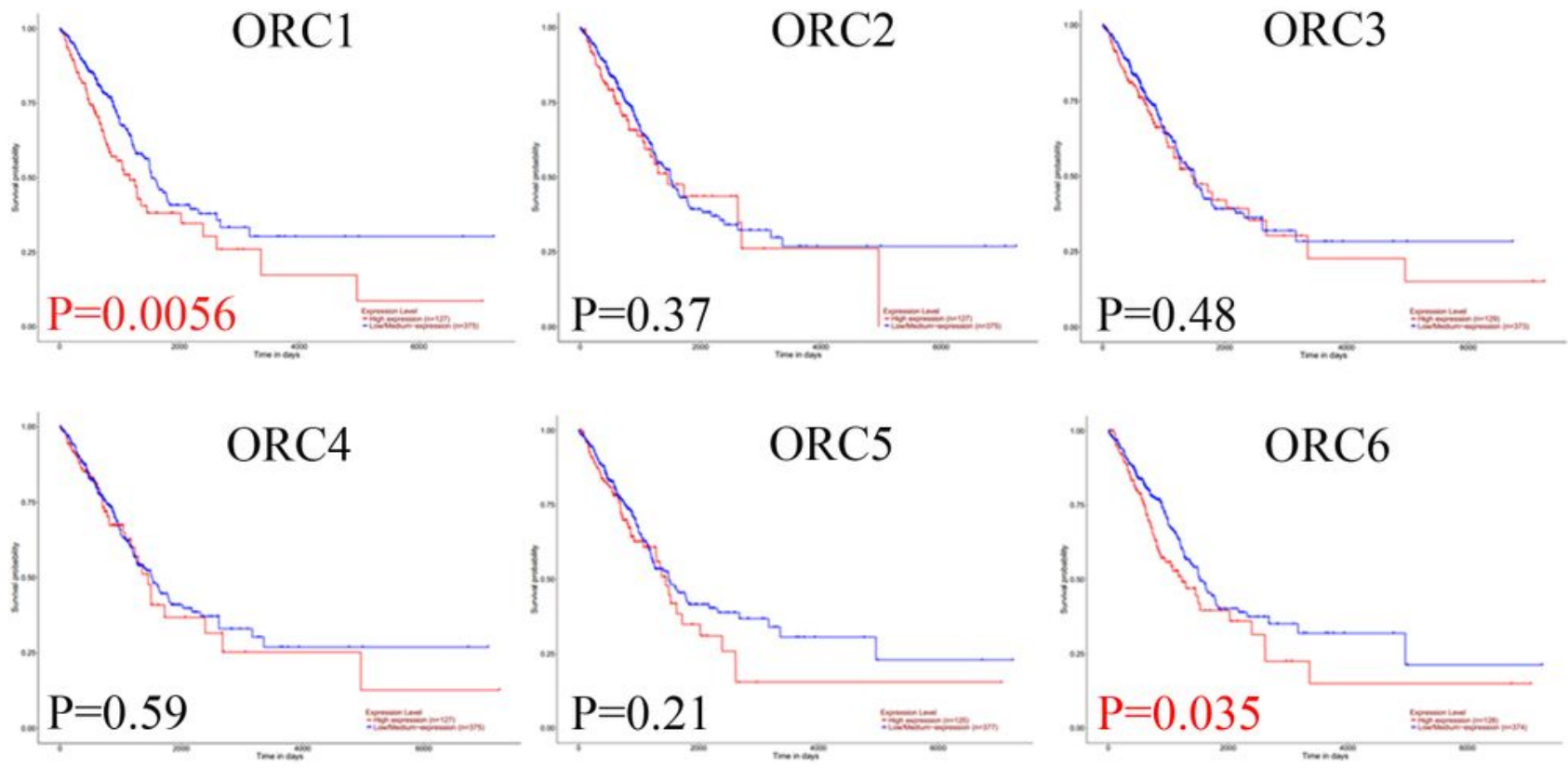

Figure 3

ORC complex mRNA level in LUAD based on UALCAN database. A) ORC1-6 level in LUAD compared to normal lung samples based on UALCAN database. B) the prognostic significance of ORC1-6 mRNA in LUAD patients. ${ }^{\star \star *} p<0.001$ 
A
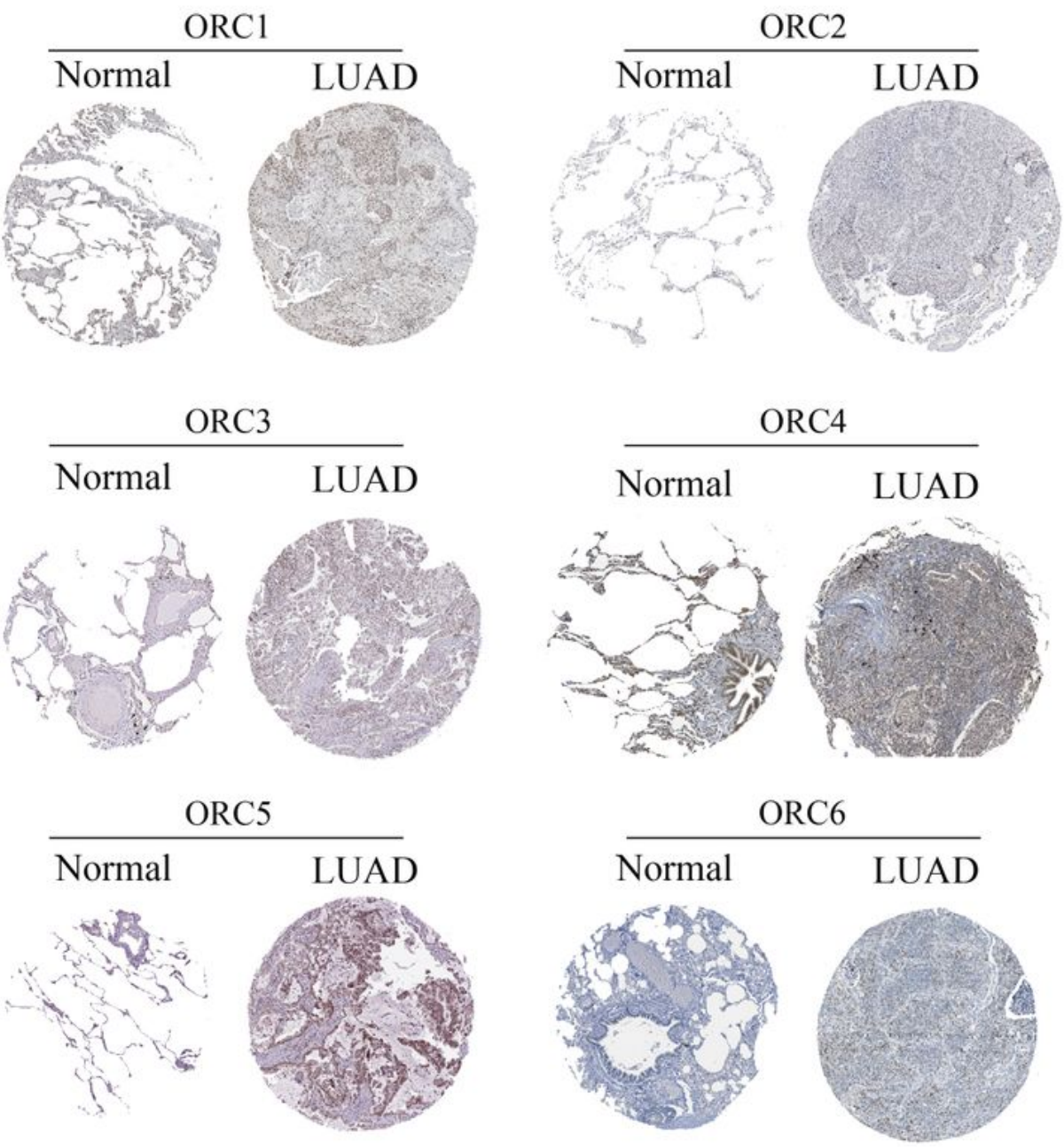

B
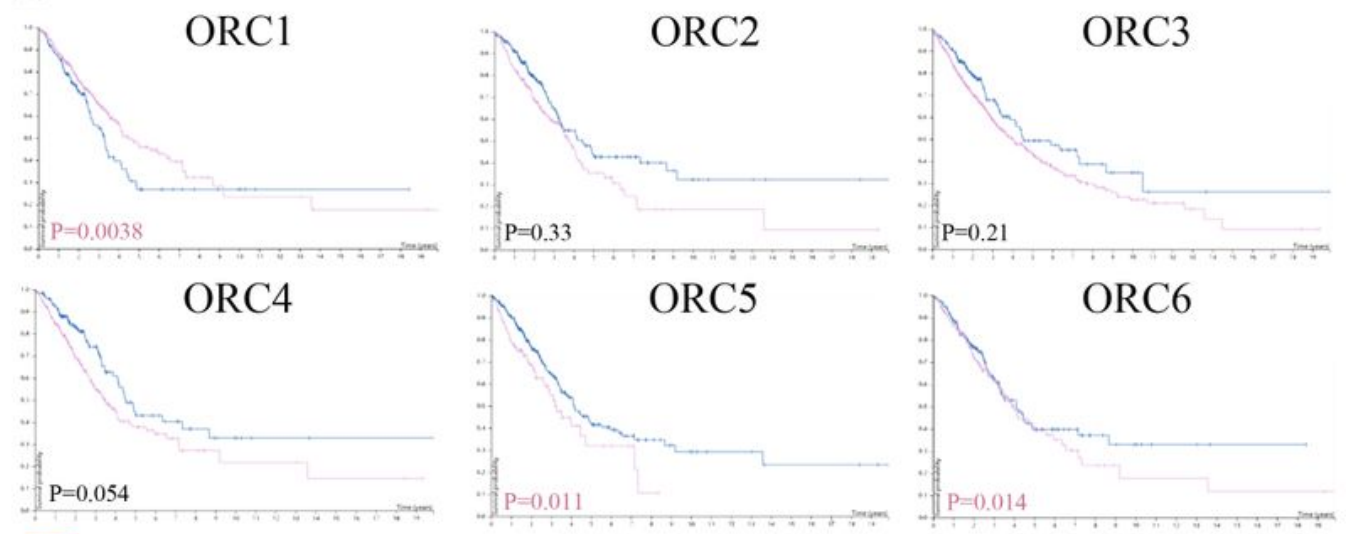

Low expression

High expression

\section{Figure 4}

ORC proteins expression in LUAD based on HPA database. A) The expression of ORC protein in LUAD compared to normal lung samples based on HPA database. B) the prognostic significance of ORC1-6 protein level in LUAD patients. 
A
ত
0
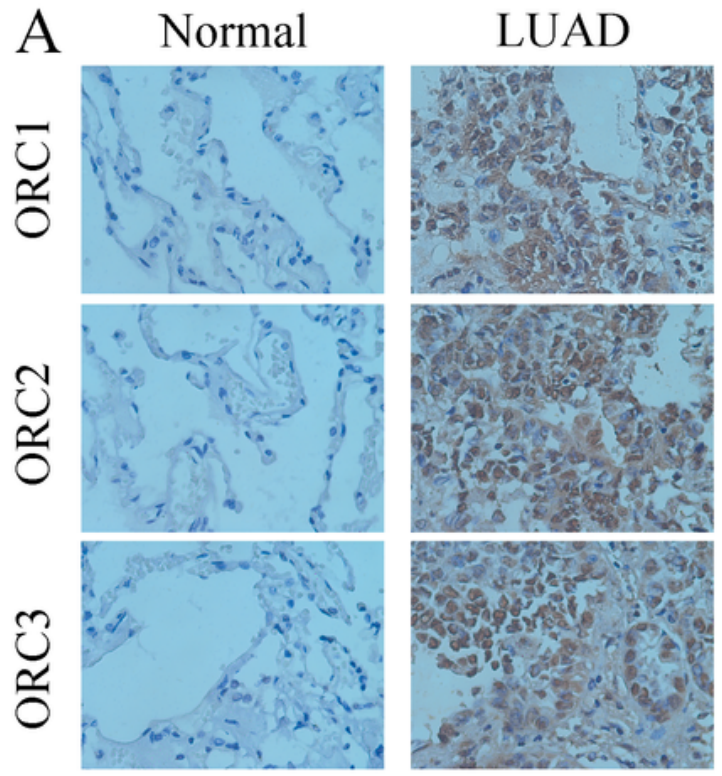
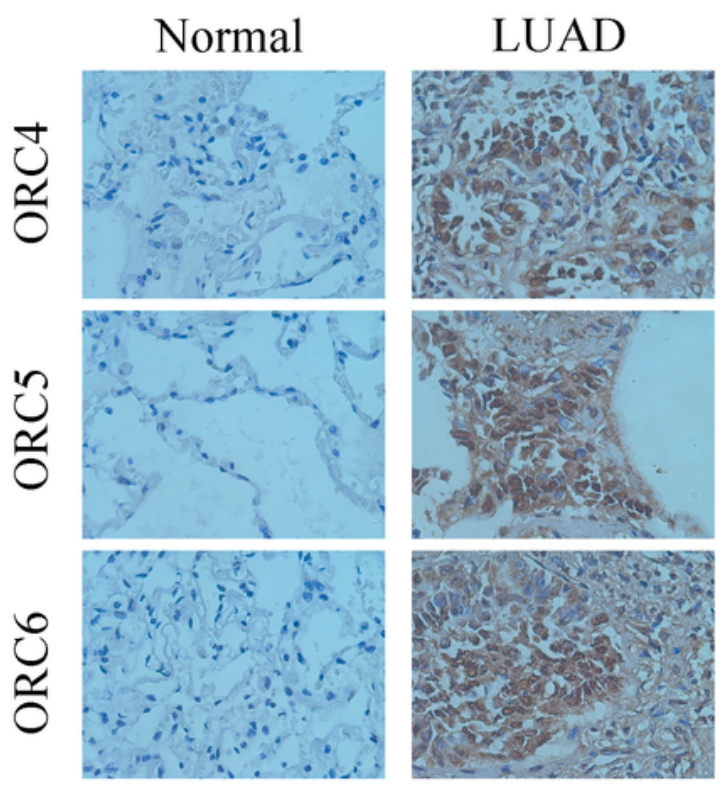

\section{B}

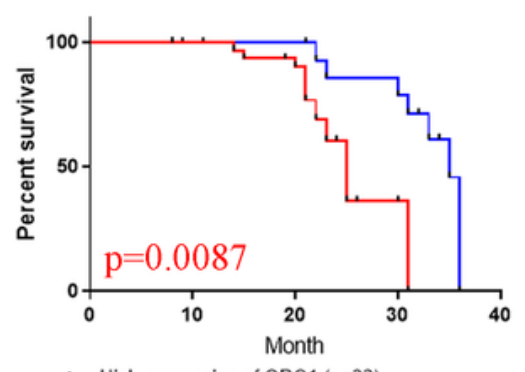

- High expression of ORC1 ( $n=32)$

- Low expression of ORC1 $(n=18)$

ORC4

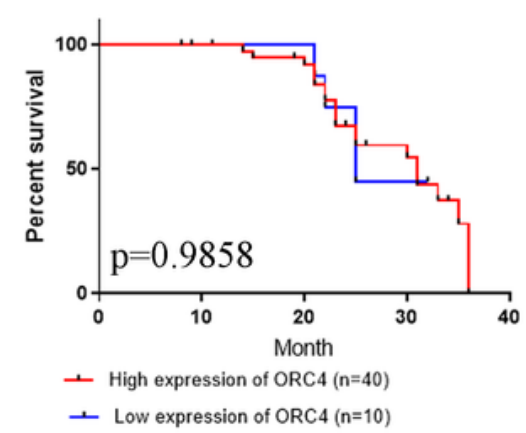

ORC2

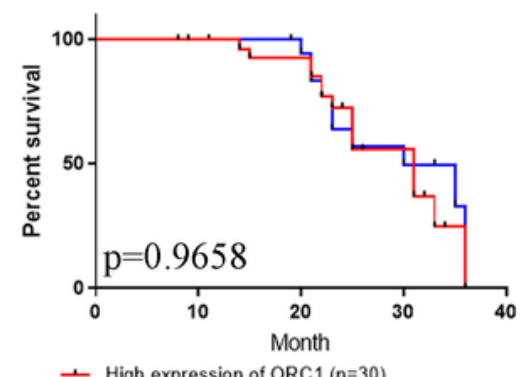

- High expression of ORC1 $(n=30)$

+ Low expression of ORC1 $(n=20)$

ORC5

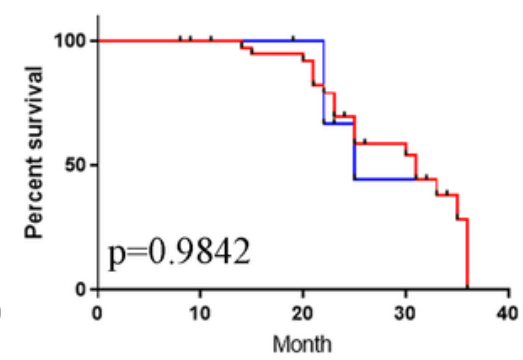

- High expression of ORC5 ( $(n=43)$

- Low expression of ORC5 (n=7)
ORC3

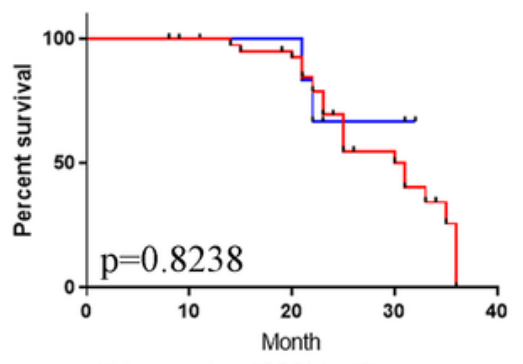

- High expression of ORC3 $(n=42)$

- Low expression of $\mathrm{ORC} 3(\mathrm{n}=8)$

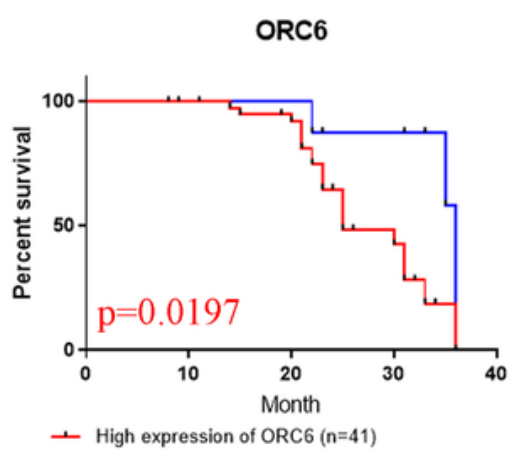

- High expression of ORC6 $(n=41)$

- Low expression of ORC6 $(\mathrm{n}=9)$

\section{Figure 5}

ORC proteins expression in LUAD by IHC staining. A) The level of ORC in LUAD compared to normal lung samples. B) the prognostic values of ORCs level in LUAD patients. 

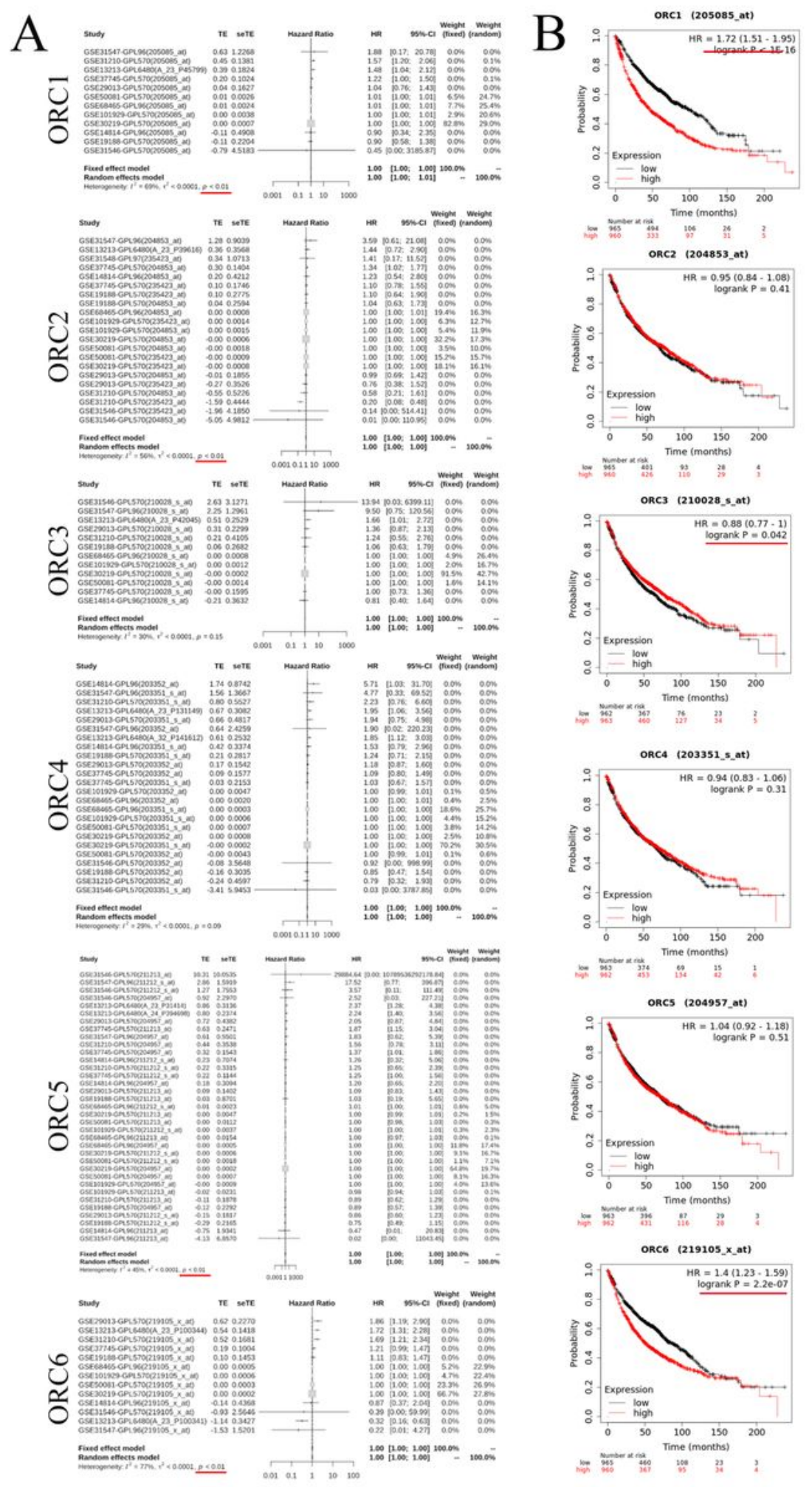

\section{Figure 6}

GENT2 Meta-survival analysis and Kaplan-Meier Plotter survival analysis. A) The meta-survival analysis for ORCs based on GENT2 database. (B) The survival analysis of ORCs based on the KM-Plotter database. 


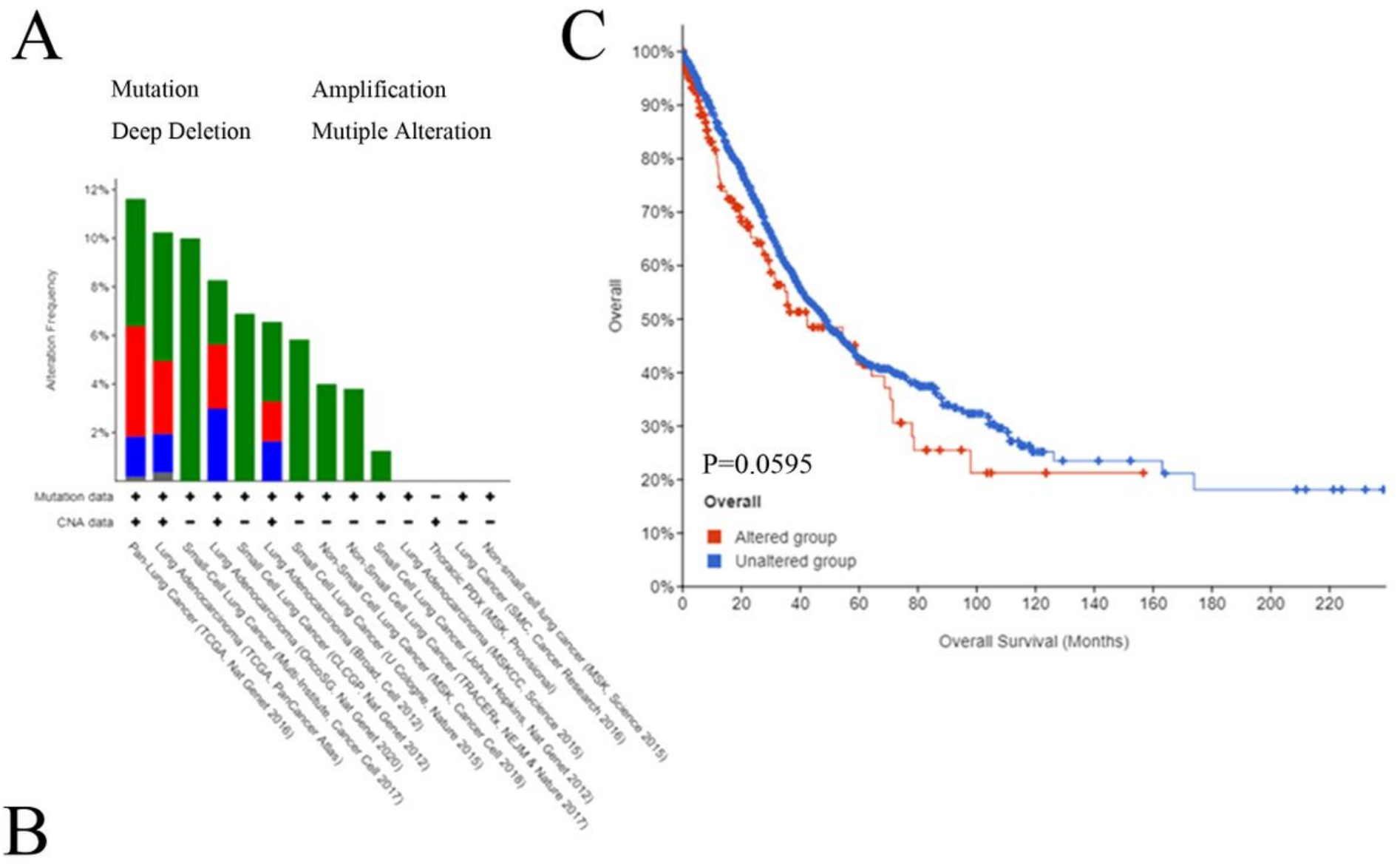

ORC1 $1.9 \%$ * In|-||||||||ORC2 $1.7 \%$.

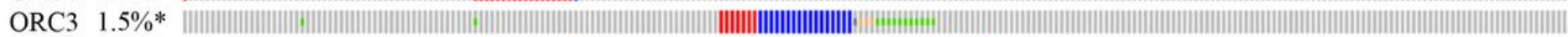
ORC4 $1.2 \% *$ |

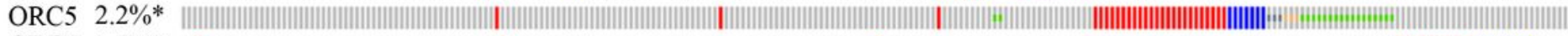

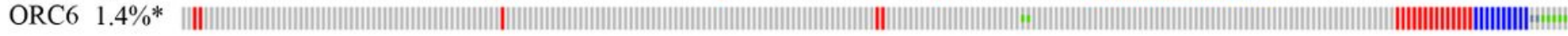

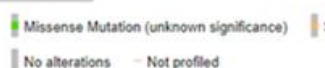

if Truncating Muration (uninown significance)

Amplifcaton (unkinown significance)

I Deep Delesson (uninown significance)

\section{Figure 7}

The alteration of ORCs. A) The DNA alteration of ORCs in LUAD. B) The frequency of ORC complex in LUAD based on the cBioProtal database. C) OS in LUAD patients with or without ORC alteration. 
A
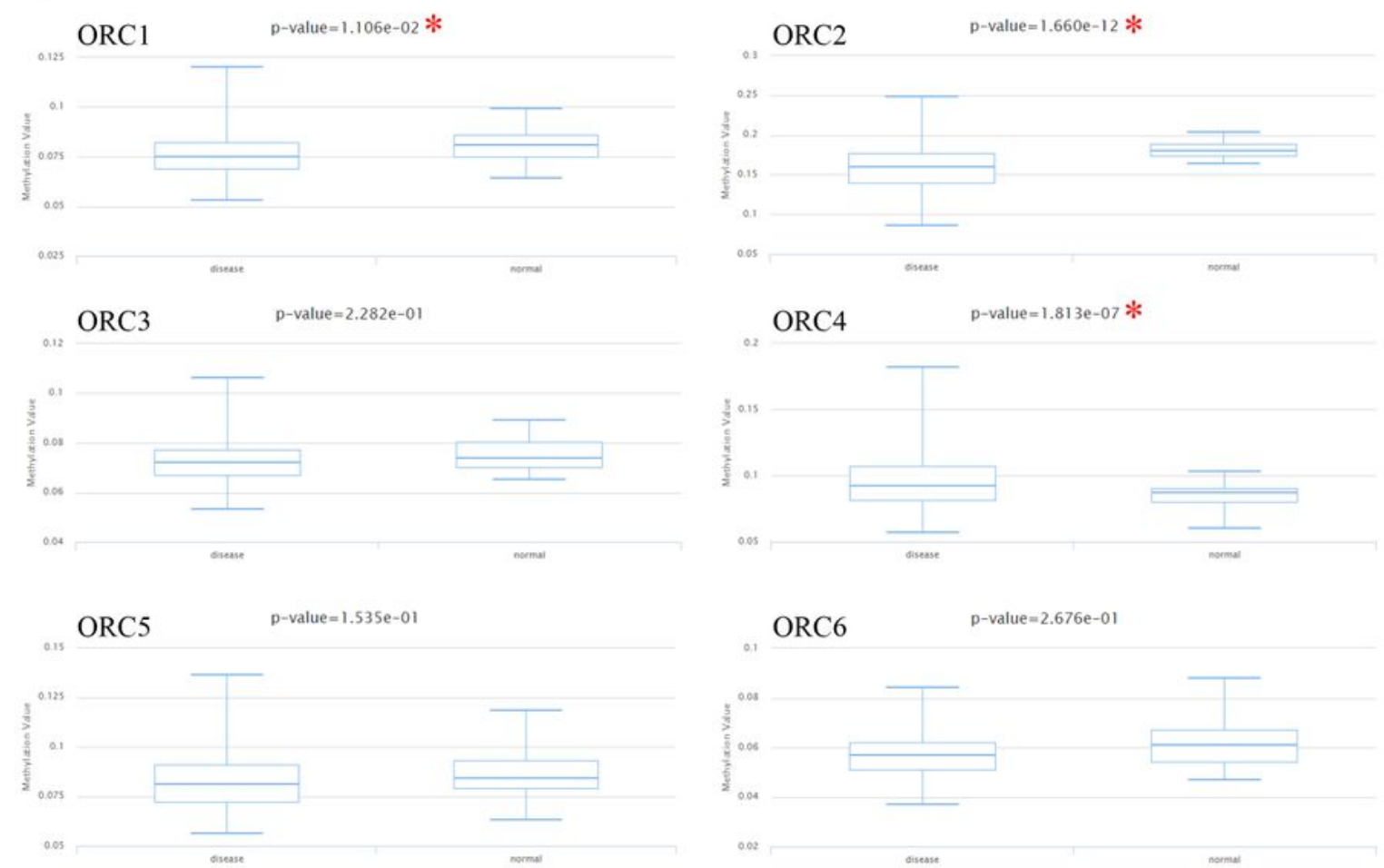

B
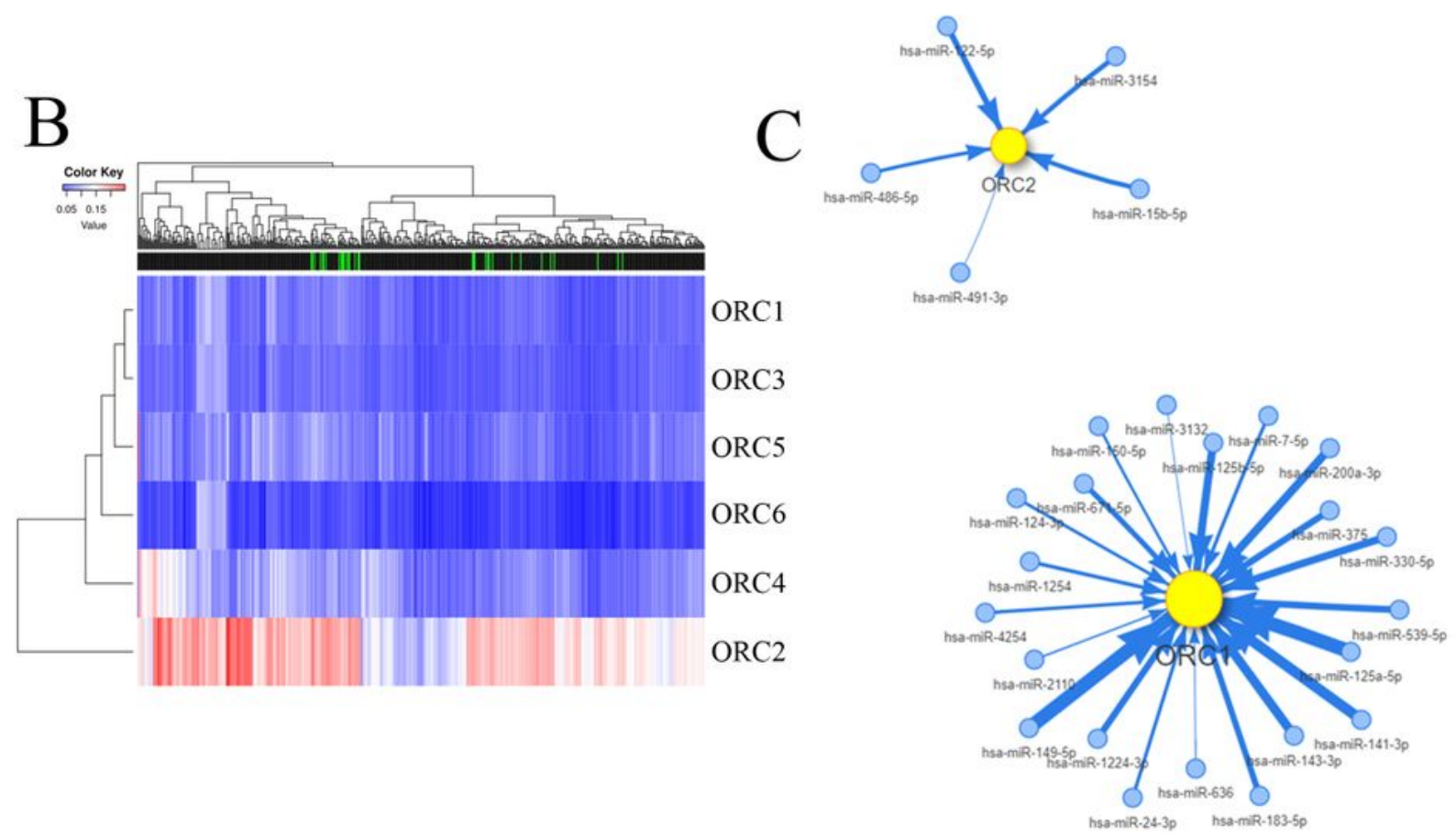

\section{Figure 8}

DNA methylation and miRNA network of ORCs. A) The DNA methylation of ORCs in LUAD compared to normal lung samples based on TCGA database. B) The heat map for ORC methylation in LUAD and normal lung samples. C) The miRNA network of ORCs. 


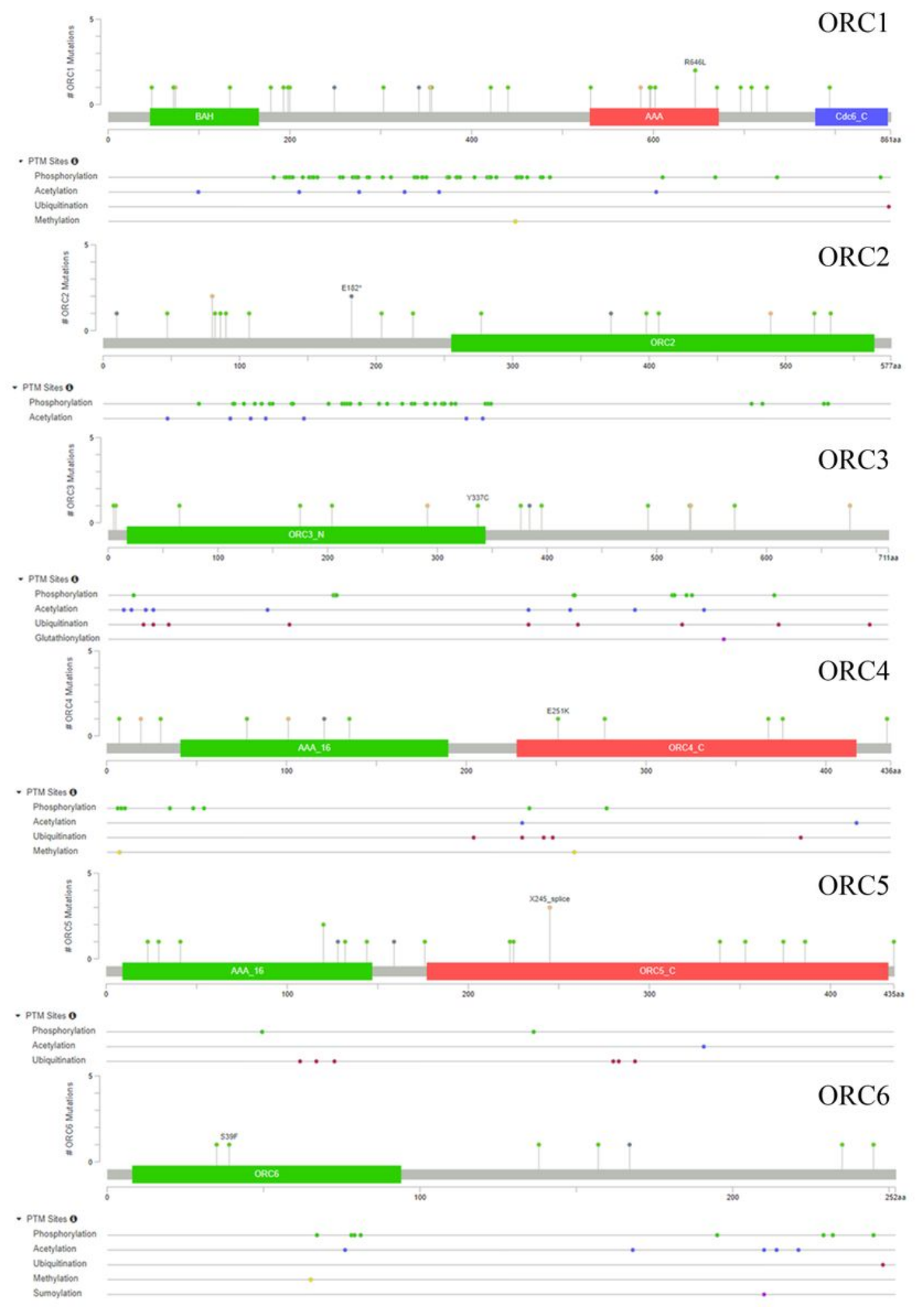

Figure 9

The protein secondary structure of ORC1-6. 
A
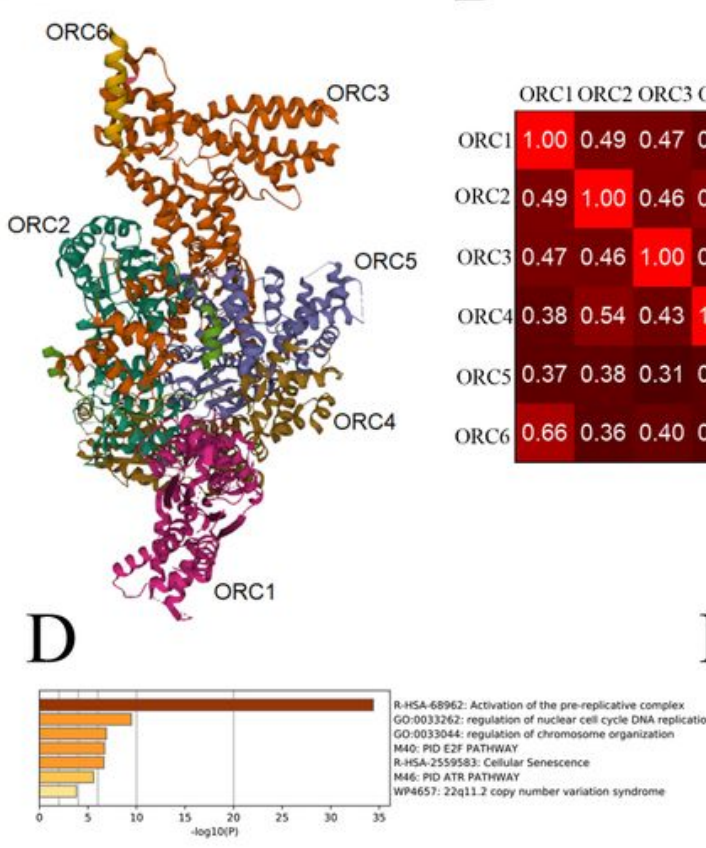

$\mathrm{F}$

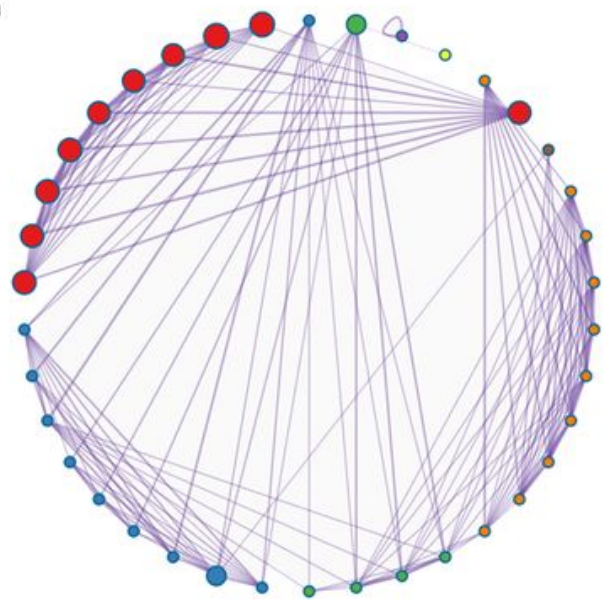

B C

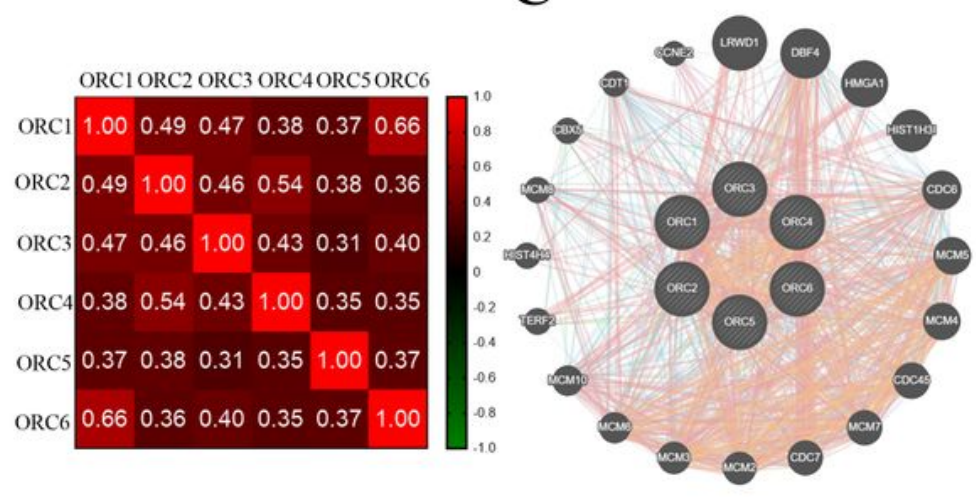

$\mathrm{E}$

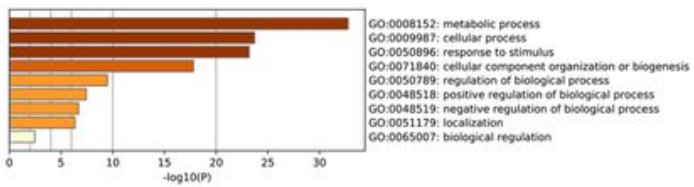

$\mathrm{G}$

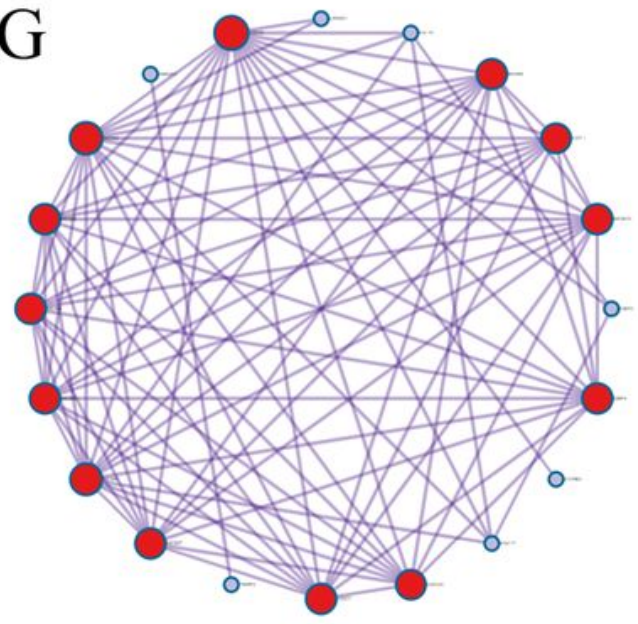

Activation of the pre-replicative complex

- regulation of nuclear cell cycle DNA replication

- regulation of chromosome organization

DID E2F PATHWAY

Cellular Senescence

PID ATR PATHWAY

E22q11.2 copy number variation syndrome

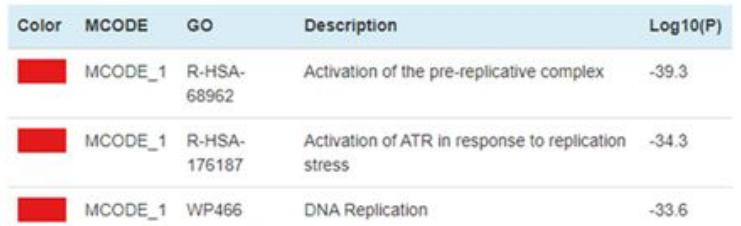

33.6

\section{Figure 10}

The co-expression, interaction, function enrichment and KEGG pathway enrichment. A) the tertiary structure of ORCs. B) Spearman's correlation analysis of ORCs. C) The protein-protein interaction network among ORCs. D) Pathway and process enrichment analysis. E) The top-level Gene Ontology biological processes. F) The networks for pathway and process enrichment analysis. G) The networks for proteinprotein interaction enrichment analysis. 


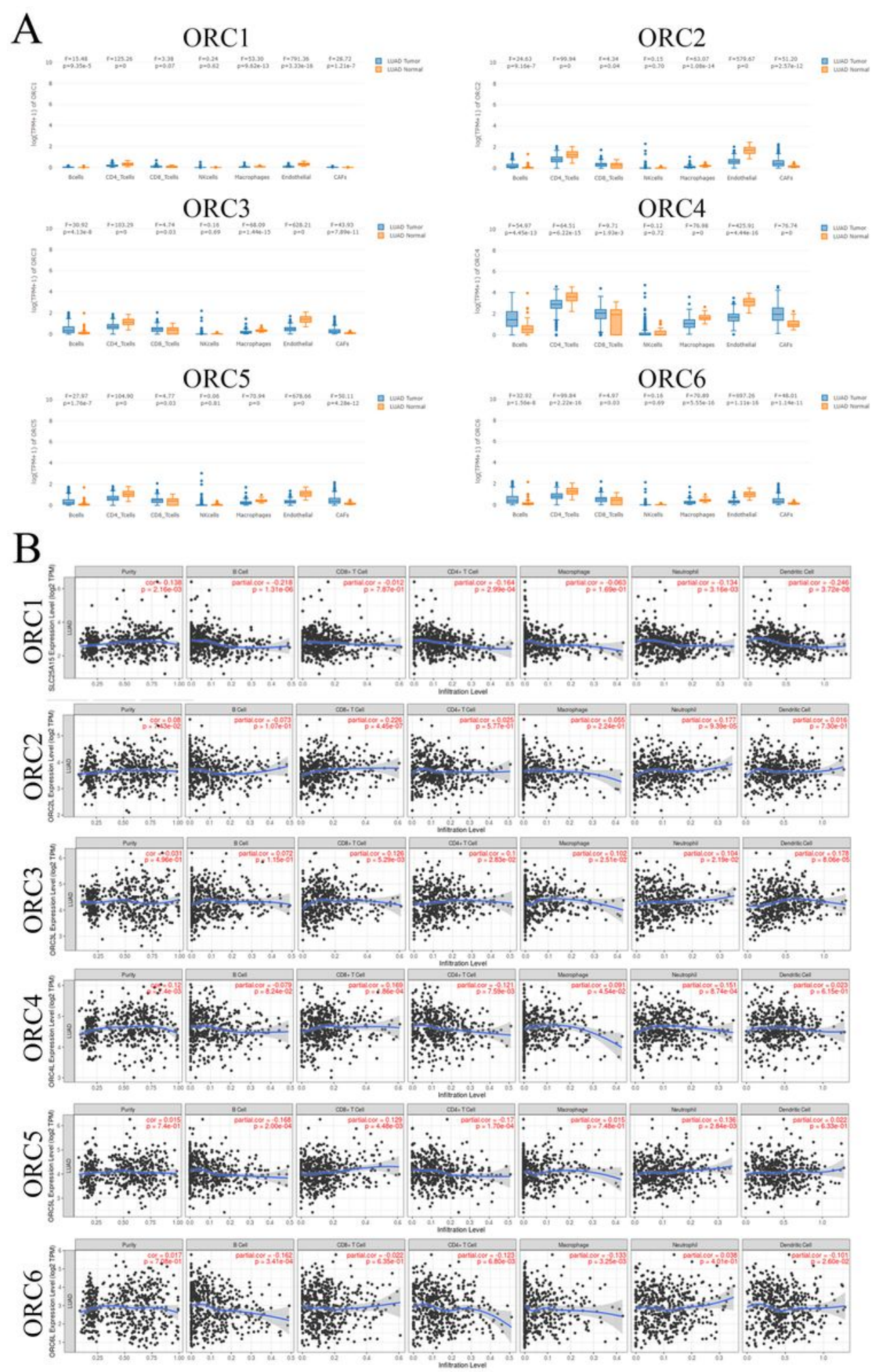

\section{Figure 11}

Immune infiltration of ORC complex in LUAD. A) The ORCs expression in each immune cell type, and perform the cell type-level differential expression analysis. B) Cancer purity and immune infiltration.

\section{Supplementary Files}


This is a list of supplementary files associated with this preprint. Click to download.

- SupplementaryFiguresS1.jpg

- SupplementaryFiguresS2.jpg 